\title{
High thermoelectric performance in two-dimensional tellurium: An ab initio study
}

\author{
Zhibin Gao, ${ }^{\dagger}$ Gang Liu, ${ }^{*, \dagger}$ and Jie Ren*,† \\ $\dagger$ Center for Phononics and Thermal Energy Science, China-EU Joint Center for Nanophononics, \\ Shanghai Key Laboratory of Special Artificial Microstructure Materials and Technology, School \\ of Physics Sciences and Engineering, Tongji University, Shanghai 200092, China \\ $\ddagger$ School of Physics and Engineering, Henan University of Science and Technology, Luoyang \\ 471023, China \\ E-mail: liugang8105@gmail.com; Xonics@tongji.edu.cn
}

\section{Abstract}

In 2016, bulk tellurium was experimentally observed as a remarkable thermoelectric material. Recently, two-dimensional (2D) tellurium, called tellurene, has been synthesized and has exhibited unexpected electronic properties compared with the $2 \mathrm{D} \mathrm{MoS}$. They have also been fabricated into air-stable and high efficient field-effect transistors. There are two stable 2D tellurene phases. One $(\beta-\mathrm{Te})$ has been confirmed with an ultralow lattice thermal conductivity $\left(\kappa_{L}\right)$. However, the study of the transport properties of the other more stable phase, $\alpha$-Te, is still lacking. Here, we report the thermoelectric performance and phonon properties of $\alpha$-Te using Boltzmann transport theory and first principle calculations. A maximum $Z T$ value of 0.83 is achieved under reasonable hole concentration, suggesting that the monolayer $\alpha$-Te is a potential competitor in the thermoelectric field.

\section{Keywords}

tellurene, stability, Seebeck coefficient, strong anharmonicity, thermoelectric material

\section{Introduction}

Recently, 2D materials have triggered a large number of interest due to the striking physical properties related to the low dimensionality. 1. 3 Graphene ${ }^{1}$ in group-IV, borophene in group-III ${ }^{4 / 5}$ and black phosphorous in group- $\mathrm{V}^{6 / 7}$ have been extensively investigated experimentally and theoretically. However, researches related to group-VI monolayer materials are still lacking to date.

Recently, Zhu et al. ${ }^{8}$ firstly have proposed three novel types of monolayer tellurene, namely $\alpha$-, $\beta$ - and $\gamma$-Te. Afterwards, tellurene has been synthesized and attracted lots of interest. ${ }^{8-15}$ These findings indicate that tellurene possesses unusually physical properties, especially for applications in electronic and thermoelectric devices. Furthermore, Wang et al. ${ }^{10}$ pointed out that tellurene can be obtained in a very large scale and can be fabricated into air-stable and high-performance field-effect transistors, which makes it different from other 2D materials. Furthermore, tellurene has much larger carrier mobility than that of $2 \mathrm{D}$ $\mathrm{MoS}_{2} \cdot{ }^{8}$ Few layer tellurene also has extraordinarily electronic transport properties. 10 14]15

$\beta$-Te has the intrinsic $\kappa_{L}$ of 2.16 and $4.08 \mathrm{~W} / \mathrm{mK}$ along both directions $(300 \mathrm{~K})^{\frac{12}{2}}$ and also shows significant anisotropy, indicating good potential in application of thermoelectric devices with $Z T=$ 0.8 16 Besides, in 2016, bulk tellurium was experimentally observed as a remarkable thermo- 
electric material. ${ }^{17}$ Energetically, monolayer $\alpha$-Te is the most stable structure compared with the metastable $\beta$ - and $\gamma$-Te phases. ${ }^{8}$ As the most stable phase, $\alpha$-Te is also a promising one to be synthesized experimentally and has applications in practical nanoelectronics.

Generally, we use $Z T$ to assess the degree of thermoelectric material by $Z T=$ $S^{2} \sigma T /\left(\kappa_{e}+\kappa_{L}\right)$, where $S$ means Seebeck coefficient, $\sigma$ is the electric conductivity, $T$ is the absolute temperature, $\kappa_{e}$ and $\kappa_{L}$ are the electronic and lattice thermal conductivity, respectively. Ideally, one would like to enhance the numerator and weaken the denominator at the same time. Fortunately, one can enhance the scattering of phonons by boundary, defect, isotopic effect, resonant bonding, ${ }^{18}$ rattle-like scattering, 19 lone electron pairs, 20 making composite structures ${ }^{21}$ and even forming solid solutions. ${ }^{22}$ Unfortunately, $S$ and $\sigma$ unusually interweave and behave in an opposite trend, which induces the complexity to efficiently realize the waste heat recovery. 21

Interestingly, low dimensional such as 2D materials have the potential to break above relation between $\mathrm{S}$ and $\sigma$ due to the quantum confinement effect pointed by Dresselhaus et al ${ }^{\sqrt{3}}$ There are three phases of atomically thin tellurium, namely $\alpha-, \beta$ and $\gamma$-Te. The first two are energetically, dynamically and mechanically stable. Furthermore, $\alpha$ Te is more stable than $\beta-\mathrm{Te}^{\sqrt{8}}$ that has an unusually low $\kappa_{L}{ }^{12}$ and superior thermoelectric property. 16 However, relevant works about $\kappa_{L}$ and thermoelectric properties of the more stable $\alpha$-Te phase is still lacking. In this letter, we focus on the more stable phase, $\alpha$-Te, and would like to explore its novel electronic and thermal properties. We find a largest $Z T(0.83)$ is achieved under reasonable hole concentration at $700 \mathrm{~K}$ for $\alpha$-Te.

\section{Computational methods}

We use vasp code and PBE functional. $23-25$ The cutoff energy is $500 \mathrm{eV}$. In order to obtain a more accurate electronic band structure and density of states, we use HSE06 approach. ${ }^{26}$ The van der Waals (vdW) correction proposed by Grimme ${ }^{27}$ was taken into consideration in our calculations. The criterion of convergence energy and force are
$10^{-6} \mathrm{eV}$ and $1 \mathrm{meV} / \AA$. We use $17 \times 17 \times 1 \mathrm{k}$-mesh in the Brillouin zone.

In the framework of Boltzmann transport theory (BTE), the electrical transport properties, such as $\sigma, S$ and $\kappa_{e}$ can be expressed as: $\underline{.28 \mid 29}$

$$
\begin{gathered}
\mathbf{K}_{n}=\frac{1}{4 \pi^{3}} \sum_{i, \mathbf{k}} \tau_{i}(\mathbf{k}) \mathbf{v}_{i}(\mathbf{k}) \otimes \mathbf{v}_{i}(\mathbf{k})\left[\varepsilon_{i}(\mathbf{k})-\mu\right]^{n} \\
\times\left[-\frac{\partial f\left(\mu, T, \varepsilon_{i}\right)}{\partial \varepsilon_{i}}\right] \\
\sigma=e^{2} \mathbf{K}_{0} \\
S=\frac{1}{e T} \mathbf{K}_{1} \mathbf{K}_{0}^{-1} \\
k_{e}=\frac{1}{T}\left(\mathbf{K}_{2}-\mathbf{K}_{1}^{2} \mathbf{K}_{0}^{-1}\right)
\end{gathered}
$$

where $\tau_{i}(\mathbf{k})$ and $\varepsilon_{i}$ are the electronic relaxation time and energy eigenvalue. $k_{e}$ can be obtained based on the tensor $\mathbf{K}_{n}$.

The in-plane $\kappa_{L}$ under relaxation time approximation can be obtained:12

$$
\kappa_{\alpha \beta}=\frac{1}{V} \sum_{\lambda} C_{\lambda} v_{\lambda \alpha} v_{\lambda \beta} \tau_{\lambda},
$$

where the meaning of each parameter are explained elsewhere. $\frac{12}{}$ We use Phonopy ${ }^{30}$ and ShengBTE ${ }^{31}$ to deal with the harmonic and anharmonic force constants. We use the equation (4) to calculate the electronic thermal conductivity $k_{e}$ since sometimes the results from the Wiedemann-Franz law is unreasonable, such as in metallic $\mathrm{VO}_{2},{ }^{32}$ clean graphene near the charge neutral point, ${ }^{33}$ and single-electron transistor. ${ }^{34}$ As a matter of fact, the WiedemannFranz law is only suitable for the system where the scattering of electrons in the material is dominanted by the elastic collision ("good metal") ${ }^{35}$ Therefore, we use the definition to calculate $k_{e}$. It should be noted that an effective thickness should be defined in order to calculate the electronic and thermal properties for 2D materials. The thickness of $\alpha$-Te is $7.74 \AA$, whose definition is clearly defined in elsewhere. $12[36$ 


\section{Results and discussion}

The optimized structure of $\alpha$-Te is shown in Figure $1 \mathrm{a}$ and $1 \mathrm{~b}$ with lattice constant $4.238 \AA$ obtained by $\mathrm{PBE}+\mathrm{D} 2$ functional. The $\alpha$-Te possesses $P$ 3M1 (164) symmetry group belonging to trigonal system and isotropic pattern in 2D plane, which is quite different from the anisotropic bulk $\mathrm{Te}^{37}$ and $2 \mathrm{D} \beta$-Te.${ }^{8.12}$ From the top and side views, $\alpha$ Te and sandwiched $1 \mathrm{~T}-\mathrm{MoS}_{2}$ look alike in many ways. Intermediate Te atom is octahedrally coordinated to six neighboring Te atoms and upper and lower Te layers form the A-B like stacking. The coordination numbers in outer and centered Te are 3 and 6, which is the characteristic of a multivalency formation of Te atom located in the near bottom of the periodic table. The bond length in $\alpha$-Te is all $3.04 \AA$, which is larger than those of $2.77 \AA$ and $3.03 \AA$ (two type bonds) in monolayer $\beta$-Te. 12

Bulk Te was reported a direct semiconductor $\left(\mathrm{E}_{g}\right.$ $=0.25 \mathrm{eV})^{37}$ and it also has recently been experimentally observed as a remarkable thermoelectric material. ${ }^{17}$ Figure $1 \mathrm{c}$ and 1d show the electronic band structure and density of states (DOS) of $\alpha$ Te, indicating a near-direct band gap material $\left(\mathrm{E}_{g}\right.$ $=1.11 \mathrm{eV}$ ) at HSE06 level. Our result is very consistent with the previous theoretical work. .8

A good thermoelectric material not only needs a minimum thermal conductivity, but also requires a simultaneously maximum power factor $(P F)$. However, Seebeck $S$ and $\sigma$ are usually interweaved. A large $S$ requires a large carrier effective mass decided by $\frac{38}{38} S=\frac{8 \pi^{2} k_{B}^{2} T}{3 e h^{2}} m^{*}\left(\frac{\pi}{3 n}\right)^{2 / 3}$ in 3D semiconducting materials, but $\sigma$ is inversely proportional to the carrier effective mass due to $\mu=\frac{e \tau}{m^{*}}$.In 1993, Hicks and Dresselhaus proposed a seminal idea that sharpen the energy dependence of the DOS in 1D and 2D systems to alleviate the coupling between $S$ and $\sigma$, consequently enhancing the $P F .39940$ Mahan and Sofo further generalized it to a refined sentence. ${ }^{41}$ Afterwards, these guiding principles triggered two interesting band structure shapes. One is "pudding-mold" $42 / 43$ and the other one is "Mexican-hat-shape". ${ }^{44}$ Recently, a backward thinking emerges, which introduce quasi-one-dimensional electronic band dispersions in 2D or 3D materials to increase the thermoelectric performance. ${ }^{45 / 46}$ A relatively flat band means a large DOS in a narrow energy region near the Fermi level. The dispersive band leads to a high carrier velocity, also indicates a small $m^{*}$ and therefore a high $\mu .47-50]$ Interestingly, The valence bands near the Fermi level of $\alpha$-Te shown in Figure 1c has some hole pockets and relatively flat bands, which is the characteristic of a good thermoelectric property and is similar to renowned $\mathrm{PbTe}_{1-x} \mathrm{Se}_{x}$ thermoelectrics. .51

Based on the band structure, we could evaluate the $S, \sigma$, and $\tau$ of $\alpha$-Te. Since the melting point of tellurium is $723 \mathrm{~K}$, we select three typical temperatures $(300,500$ and $700 \mathrm{~K})$ in the whole calculations. Figure 2a shows the Seebeck coefficients $S$ in the n- and p-type of $\alpha$-Te as functions of carrier concentration. Overall, the absolute values of $S$ decrease for both n- and p-type when increasing the carrier concentration, which reflects the inverse proportion between $S$ and carrier concentration $n$. Moreover, the absolute value of $S$ of hole doping is bigger than the electron doping in $\alpha$-Te. For instance, the value of $|S|$ for n-type is $242.9 \mu \mathrm{V} / \mathrm{K}$ at $10^{12} \mathrm{~cm}^{-2}$ carrier concentration at $500 \mathrm{~K}$ temperature, only around half of p-type $529.6 \mu \mathrm{V} / \mathrm{K}$ at same condition. The maximum value $S$ of p-type $\alpha$-Te is around $700 \mu \mathrm{V} / \mathrm{K}$ at room temperature, which is the double of that $350 \mu \mathrm{V} / \mathrm{K}$ in bulk tellurium. ${ }^{17}$ This is the physical reason that the $Z T$ of $\alpha$-Te are comparable with bulk tellurium, although the $\kappa_{L}$ in $\alpha$-Te $(9.85 \mathrm{~W} / \mathrm{mK})$ is around 7 times of that in bulk tellurium $(1.5 \mathrm{~W} / \mathrm{mK})$. 17 In this sense, if one further increases the phonon scattering using some defects and isotope effect in $\alpha$-Te, the $\kappa_{L}$ will decrease but with the same level of Seebeck coefficient $S$. Then the $Z T$ of $\alpha$-Te will be significantly enhanced and surpass the bulk tellurium. 17

The difference between electron and hole doping in Seebeck $S$ originates from the electronic band structure and DOS in Figure 1c and 1d. As we discussed above, $S$ is primarily regulated by the effect mass $\mathrm{m}^{*}$ and the magnitude of DOS. A more smooth valence band (hole-doping) compared with a conduction band (electron-doping) corresponds to a larger carrier $\mathrm{m}^{*}$, indicating a large $S$ since $S$ is proportional to the $\mathrm{m}^{*}$. Furthermore, DOS of p-type is obviously much larger than n-type of $\alpha$-Te around the Fermi level shown in Figure 1d. These evidences verify that the superior performance of the hole doping compared 
with the electron doping in $\alpha$-Te.

According to the BTE, the electronic conductivity $\sigma$ is dependent and is proportional to the relaxation time $\tau$. Therefore, a reasonable relaxation time $\tau$ should be chosen. As a matter of fact, $\tau$ in materials is a function of temperature and carrier concentration. So far, experimental measurement may be the only effective way to solve this issue. For theoretical calculations, $\tau$ is difficult to obtain with high precision. Specifically, there are mainly three methods, such as using a designated $\tau$ based on revelent experiments, $\underline{5253}$ electro-phonon coupling implemented in EPW. ${ }^{54}$ Another approach is based on the well-known model: $\tau=\frac{m^{*} \mu}{e}$ in which $\mu$ is the carrier mobility and in $2 \mathrm{D}$ materials using deformation potential theory acoustic phonon limited $\mu$ could be expressed as: $\underline{.55}$

$$
\mu_{2 D}=\frac{e \hbar^{3} C_{2 D}}{k_{B} T m^{*} m_{d} E_{i}^{2}}
$$

where $\mathrm{m}_{d}$ is the average effective mass dominated by $\mathrm{m}_{d}=\sqrt{m_{x}^{*} m_{y}^{*}} . E_{i}$ can be expressed as $E_{i}=$ $\Delta E_{i} /\left(\Delta l / l_{0}\right)$ in which $\Delta E_{i}$ is the $i^{t h}$ band under small compression and expansion compared with the energy of unstrained system. Here, it supposes that the energy of core electrons, considered as the energy reference, do not change under the small deformation. This treating process is reasonable and have also been verified by the previous researches. ${ }^{45[55]}$ The detailed calculations is shown in the Supporting Information. Our calculated effective masses $m^{*}$ are $0.107 m_{e}$ for the conduction band minimum and $0.164 m_{e}$ for the valence band maximum and the carrier mobilities are $2.086 \times 10^{3} \mathrm{~cm}^{2} \mathrm{~V}^{-1} \mathrm{~s}^{-1}$ for electrons and $1.736 \times 10^{3} \mathrm{~cm}^{2} \mathrm{~V}^{-1} \mathrm{~s}^{-1}$ for holes. Our result is very consistent with the previous report. $\frac{8}{1}$ Therefore, based on $\tau=\frac{m^{*} \mu}{e}$, we can obtain the relaxation time of 0.127 ps for electrons and 0.163 ps for holes in $\alpha$-Te.

Contrary to the Seebeck $S$, electronic conductivity $\sigma$ increases with increasing carrier concentration. Figure 2b shows a larger $\sigma$ of electron doping compared with hole doping at the same carrier concentration due to the smaller effective mass $\left(0.107 \mathrm{~m}_{e}\right.$ for electrons $<0.164 \mathrm{~m}_{e}$ for holes $)$. Furthermore, $\sigma$ of two type $\alpha$-Te are insensitive to temperature, which is similar to that of $\beta$-Te. 16
A high $P F$ needs a large $S$ and a large $\sigma$ simultaneously. One should compromise between decreasing function of $S$ and increasing function of $\sigma$. The calculated power factor $\left(S^{2} \sigma\right)$ that can demonstrate the thermoelectric performance of $\alpha$ Te is shown in Figure 2c. The $P F$ for both types first increase, climb the summit at moderate carrier concentrations, and then decrease when increasing the concentration. The p-type $P F$ is significantly larger than n-type of $\alpha$-Te, though they exhibit similar trend dependent on the carrier concentration. For instance, the maximum $P F$ of holes and electrons are $74.6 \mathrm{~mW} / \mathrm{mK}^{2}$ at $6.12 \times 10^{13}$ concentration and $21.2 \mathrm{~mW} / \mathrm{mK}^{2}$ at $2.86 \times 10^{13}$ concentration at $500 \mathrm{~K}$. The former one is around 2.5 times larger than that of the latter one and is 2 times larger than 2D SnSe. 56

More interestingly, the maximum $P F$ of bulk tellurium at room temperature is around $1.3 \mathrm{~mW} / \mathrm{mK}^{2}, 17$ which is around a tenth of that in $\alpha$-Te $\left(12 \mathrm{~mW} / \mathrm{mK}^{2}\right)$ at the similar condition shown in Figure 2c. The physical reason behind it is the very small effective mass $m^{*}$ of $\alpha$-Te. The $m^{*}$ of standard $2 \mathrm{H}-\mathrm{MoS}_{2}$ is $0.47 m_{e}$ for electrons and $0.58 m_{e}$ for holes. ${ }^{8}$ However, in $\alpha$-Te, the $m^{*}$ is $0.107 \mathrm{~m}_{e}$ for electron and $0.164 m_{e}$ for holes. Obviously, the effective mass of carriers in $\alpha$-Te is quite smaller than $2 \mathrm{H}-\mathrm{MoS}_{2}$. A smaller $m^{*}$ will result in a larger electronic conductivity qualitatively decided by the simple model: $\sigma=\frac{n e^{2} \tau}{m^{*}}$. Moreover, Liu et al. ${ }^{14}$ pointed out that lone-pair electrons in bulk Te will significantly enhance the interchain electronic transport. Similarly, the coordination number in $\alpha$-Te is 3 for outer Te atoms, which is only half of valence electrons in Te atoms (group-VI). Therefore, each outside Te atom exists 3 lone-pair electrons. These lone-pair electrons will enhance the hopping term between electrons and contribute to the superior electronic transport properties in $\alpha$-Te. .814

An optimal $Z T$ also needs a minimum thermal conductivity $\left(\kappa_{e}+\kappa_{L}\right)$. For electronic thermal conductivity, $k_{e}$ has been discussed in the computational methods. Next, we would like to explore the phonon transport properties of $\alpha$-Te, as phonon dominates the thermal transport in semiconductors. The calculated phonon dispersions are shown in Figure 3a. Firstly, it shows that all phonon branches are free from negative and this result con- 
firms the dynamical stability of $\alpha$-Te. Moreover, there is no phonon gap between acoustic and optical branches, indicating a strong optical-acoustic phonon scattering which will suppress the $\kappa_{L}{ }^{57}$ of materials.

A high-order $(\geq 3)$ scattering of phonons leads to a limited $\kappa_{L} \cdot{ }^{5859}$ Figure $3 \mathrm{~b}$ shows the intrinsic $\kappa_{L}$ computed with both non-iterative and iterative method from $100 \mathrm{~K}$ to $700 \mathrm{~K}$. We note that both methods give similar trends of $\kappa_{L}$ in the whole temperature range. However, the iterative method always gives values of $\kappa_{L}$ higher than the non-iterative method at the same temperature. We choose the values of iterative method in the following discussion. We find $\kappa_{L}$ of $\alpha$-Te can be well described by the $T^{-1}$ curve. This phenomenon is also found in bulk $\mathrm{Te}^{17]}$ and $\beta$-Te. ${ }^{[12}$ The value of intrinsic $\kappa_{L}$ is $9.85 \mathrm{~W} \mathrm{~m}^{-1} \mathrm{~K}^{-1}(300 \mathrm{~K})$, which is twice as much as that of $\beta$-Te at the same temperature. ${ }^{12}$ Furthermore, $\kappa_{L}$ of $\alpha$-Te is also larger than bulk Te $(2.77 \mathrm{~W} / \mathrm{mK}), \frac{12}{12}$ which is consistent with the familiar trend $\left(\kappa_{2 D}>\kappa_{3 D}\right)$. 12[60]61

The normalized contribution of ZA, TA, LA and optical branches varying with temperature are plotted in Figure 3c, in which each mode varies small at different temperatures especially beyond $200 \mathrm{~K}$, and can be regarded as independent of temperature, similar with the $\beta$-Te. ${ }^{12}$ At room temperature, three acoustic branches contribute about $90 \%$ to the total intrinsic $\kappa_{L}$, while the summation of all optical phonons is only $10 \%$. Furthermore, the proportions of contributions to total $\kappa_{L}$ are about $25 \%, 47 \%$, and $17 \%$ for LA, TA, and ZA phonons, in which ZA mode is the smallest one among three acoustic branches in $\alpha$-Te, quite different from graphene where the proportion of ZA mode is about $75 \%$. 62

It was reported that there is a symmetry selection rule in flat graphene that only even number of ZA modes can be involved in scattering processes. ${ }^{63,64}$ This rule originates from the reflection symmetry about $\mathrm{c}$ axis in the structure. The thermal resistance roots in the high order of phonon scattering. Due to the selection rule, ZA mode contributes mainly to the $\kappa$ in graphene. However, the lattice does not have the reflection symmetry about $\mathrm{c}$ axis in $\alpha$-Te. As a result the selection rule is broken, the importance of ZA mode in thermal transport of $\alpha$-Te is much smaller than graphene.
Moreover, it is worthwhile to explore the contribution of phonons with different frequencies to the total $\kappa_{L}$. Here the frequency-resolved $\kappa_{L}$ for $\alpha$-Te at $300 \mathrm{~K}$ is displayed in Figure 3d. It can be found that the main contribution originates from acoustic phonons with frequencies lower than $2 \mathrm{THz}$, while the contribution of optical phonons is very small. This is consistent with Figure 3c. Furthermore, the acoustic phonons with very low frequency (lower than $1 \mathrm{THz}$ ) contribute most to $\kappa_{L}$. In the following, we will find the behind reason is that phonons around $\Gamma$ point usually possess large phonon relaxation time.

All samples of material have finite size in practical experiment and device application, thus the additional boundary scattering will reduce $\kappa_{L}$, especially at nanoscale or at low temperatures. Usually, in nanostructures, $\kappa_{L}$ related to boundary scattering can be evaluated as: $12[61$

$$
\frac{1}{\tau_{\lambda}^{B}}=\frac{v_{\lambda}}{L}
$$

where $L$ is the size of material. This formula represents the situation for a completely diffusive boundary scattering of phonons. Cumulative $\kappa_{L}$ $\left(\wedge<\wedge_{\max }\right)$ can also be used to study the size effect, whose definition has been defined elsewhere. ${ }^{31}$ Figure $3 \mathrm{e}$ shows the size dependence of $\kappa_{L}$ for $\alpha$-Te with completely diffusive boundary scattering at room temperature and the cumulative $\kappa_{L}$ is displayed in Figure 3f. In Figure $3 \mathrm{e}, \kappa_{L}$ becomes lower while the sample size getting smaller, as the boundary scattering becomes larger. For a specifical example, when the sample size is $1 \mu \mathrm{m}$, $\kappa_{L}$ can be suppressed to $7.56 \mathrm{~W} \mathrm{~m}^{-1} \mathrm{~K}^{-1}$, about $78 \%$ of value for the infinitely large system. When the sample size is $10 \mu \mathrm{m}, \kappa_{L}$ reaches $91 \%$ of the infinitely large system. It implies the size effect always keeps till about $10 \mu \mathrm{m}$, such as $2 \mathrm{D} \mathrm{WTe}_{2} . \underline{65}$

The size effect can also be estimated from the cumulative $\kappa_{L}$ as a function of MFP shown in Figure $3 \mathrm{f}$. We can find that the MFP range of phonons that contribute significantly to $\kappa_{L}$ is about $10 \mathrm{~nm}$ to $10 \mu \mathrm{m}$, which is consistent with our previous result in Figure 3e. In order to obtain the representative MFP, we fit cumulative $\kappa_{L}$ using a single parametric function: $\kappa\left(\wedge \leq \wedge_{\max }\right)=\kappa_{\max } /(1+$ $\left.\wedge_{0} / \wedge_{\max }\right)$, where $\kappa_{\max }$ is $\kappa_{L}$ of infinite size, $\wedge_{\max }$ 
is the maximal MFP. The fitting parameter $\wedge_{0}$ can be interpreted as the typical MFP with which the phonons contribute significantly to $\kappa_{L}$. The fitted curve is shown in Figure $3 \mathrm{f}$ by dashed line. The parameter $\wedge_{0}$ is $94 \mathrm{~nm}$, which could be interpreted as the representative MFP of $\alpha$-Te. This implies the $\kappa_{L}$ of $\alpha$-Te may decline sharply with the sample size of hundreds of $\mathrm{nm}$. This property is propitious to the electronic and thermoelectric materials based on $\alpha$-Te in the nanoscale. 10

Group velocity of phonon has great effects on thermal transport of materials, as it is proportional to $v^{2}$ based on Eq. (3). The calculated $v^{2}$ with different frequencies are shown in Figure 4a. LA branch possesses the highest $v$ among three acoustic branches, specifically more than two times of that for ZA and TA phonons. The optical phonons around $3.7 \mathrm{THz}$ has the largest $v$ but they only contribute $10 \%$ to total $\kappa_{L}$. Here $\tau$ is also displayed in Figure 4b. $\tau$ around $\Gamma$ point has the magnitude of $10^{3}-10^{4}$ ps but $\tau$ of most phonons with frequency more than $5 \mathrm{THz}$ is about $0.1-10 \mathrm{ps}$. That is the reason why phonons with very low frequency dominate the thermal transport in $\alpha$-Te (Figure 3d). Furthermore, $\tau$ of TA branch is the largest of all, about $10^{4}$ ps around the $\Gamma$ point. That is the reason why TA mode contributes most to $\kappa_{L}$ even more than LA phonons (Figure 3c). It can also explain that optical phonons contribute little to $\kappa_{L}$ since most of them have ultralow $\tau$ of only 1 to $10 \mathrm{ps}$.

Grünerisen parameter provides crucial information on the anharmonic interactions of phonons. $\gamma$ in $\alpha$-Te for whole frequency spectrum is displayed in Figure 4c. $\gamma$ of TA and LA modes are in the range of $0-2$, while those of optical modes are 1-6. Comparing with $\beta$-Te, $\gamma$ of $\alpha$ Te are smaller, indicating a lower anharmonicity and a higher $\kappa_{L} \cdot{ }^{12}$ Interestingly, it can be found $\gamma$ of acoustic and optical phonon modes have suddenly dropped around 2-2.5 THz. Since there is no acoustic-optical phonon gap, the large acousticoptical phonon scattering would lead to a giant $\gamma$ and is also the source of ultralow $\kappa_{L}$ in $\beta$-Te. ${ }^{12}$ In the three-phonon scattering process, all phonon modes must be satisfied the conservation of energy and momentum simultaneously. ${ }^{66}$ The quantitative probability of all available three-phonon scattering in $\alpha$-Te are shown in Figure $4 \mathrm{~d}$. This
$P_{3}$ phase space is one order smaller than of $\beta$-Te, indicating a weaker anharmonicity of $\alpha$-Te compared with $\beta$-Te. 12

Based on the above results, intrinsic figure of merit $Z T$ can be evaluated and exhibited in Figure 5. The climax $Z T$ at $700 \mathrm{~K}$ are 0.57 and 0.83 for electron and hole doping $\alpha$-Te, which is as high as $\beta-\mathrm{Te}^{\sqrt{16}}$ and has the same order of bulk Te. ${ }^{17}$ Room temperature ZT of n-type $\alpha$-Te is 0.54 . Our calculation suggests that monolayer $\alpha$-Te is a promising thermoelectric material. Furthermore, it can be promoted further via suppressing $\kappa_{L}$ such as grain boundary, isotope effect and vacancy. $\underline{67}$

\section{Conclusion}

In summary, we have systematically studied the extraordinarily high thermoelectric performance of monolayer $\alpha$-Te by the $a b$ initio calculations. To explain the high $Z T$, we consider the electronic and thermal transport properties. A large and asymmetric electronic density of states induce relatively flat bands, leading to a large Seebeck coefficient $S$. A small effective carrier mass $m^{*}$ of CBM (VBM) will result in a larger electronic conductivity $\sigma$. Combining both, the $P F$ in $\alpha$-Te $\left(12 \mathrm{~mW} / \mathrm{mK}^{2}\right)$ exhibits ten times as large as that in bulk tellurium $\left(1.3 \mathrm{~mW} / \mathrm{mK}^{2}\right)^{17}$ at the similar condition. For lattice thermal conductivity $\kappa_{L}, \alpha$ Te consists of heavy atomic mass, which breeds low harmonic properties, such as low group velocities. For anharmonicity, strong phonon anharmonic scattering rates including acoustic-optical phonon scattering bring about a same order of magnitude $\kappa_{L}$ of $\alpha$-Te compared with the bulk Te and $\beta$-Te. A superior electronic properties and a suitable $\kappa_{L}$ combine together leading to the ultrahigh $Z T$ in $\alpha$-Te. A maximum $Z T$ of 0.83 is achieved with reasonable hole concentration at $700 \mathrm{~K}$. Our results indicate that monolayer $\alpha$-Te is a promising thermoelectric material. We hope that $\alpha$-Te, more stable than $\beta$-Te, would be experimentally observed with high $Z T$ in the near future. 


\section{Supporting Information}

The Supporting Information is available free of charge on the ACS Publications website via the Internet at https://pubs.acs.org/journal/aamick.

Electronic band structure and effective mass around conduction band minimum and valence band maximum of two-dimensional tellurium.

\section{Author Information}

\section{Corresponding Author}

*E-mail: liugang8105@gmail . com

*E-mail: Xonics@tongji.edu.cn

\section{ORCID}

Zhibin Gao: 0000-0002-6843-381X

Jie Ren: 0000-0003-2806-7226

\section{Notes}

The authors declare no competing financial interest.

Acknowledgement We thank our member Yi Wang for helpful discussions. This work is supported by the National Natural Science Foundation of China (No. 11775159), the Natural Science Foundation of Shanghai (No. 18ZR1442800), the National Youth 1000 Talents Program in China, and the Opening Project of Shanghai Key Laboratory of Special Artificial Microstructure Materials and Technology. Computational resources have been provided by the Tongji University.

\section{References}

(1) Novoselov, K. S.; Geim, A. K.; Morozov, S. V.; Jiang, D.; Zhang, Y.; Dubonos, S. V.; Grigorieva, I. V.; Firsov, A. A. Electric Field Effect in Atomically Thin Carbon Films. Science 2004, 306, 666-669.

(2) Mounet, N.; Gibertini, M.; Schwaller, P.; Campi, D.; Merkys, A.; Marrazzo, A.; Sohier, T.; Castelli, I. E.; Cepellotti, A.; Pizzi, G.; Marzari, N. Two-Dimensional Materials from High-Throughput Computational Exfoliation of Experimentally Known Compounds. Nat. Nanotechnol. 2018, 13, 246.

(3) Dresselhaus, M. S.; Chen, G.; Tang, M. Y.; Yang, R.; Lee, H.; Wang, D.; Ren, Z.; Fleurial, J.-P.; Gogna, P. New Directions for Low-Dimensional Thermoelectric Materials. Adv. Mater. 2007, 19, 1043-1053.

(4) Tang, H.; Ismail-Beigi, S. Novel Precursors for Boron Nanotubes: The Competition of Two-Center and Three-Center Bonding in Boron Sheets. Phys. Rev. Lett. 2007, 99, 115501 .

(5) Li, D.; He, J.; Ding, G.; Tang, Q.; Ying, Y.; He, J.; Zhong, C.; Liu, Y.; Feng, C.; Sun, Q.; Zhou, H.; ; Zhou, P.; Zhang, G. StretchDriven Increase in Ultrahigh Thermal Conductance of Hydrogenated Borophene and Dimensionality Crossover in Phonon Transmission. Adv. Funct. Mater. 2018, 28, 1801685.

(6) Guan, J.; Zhu, Z.; Tománek, D. Phase Coexistence and Metal-Insulator Transition in Few-Layer Phosphorene: A Computational Study. Phys. Rev. Lett. 2014, 113, 046804.

(7) Li, L.; Yu, Y.; Ye, G. J.; Ge, Q.; Ou, X.; Wu, H.; Feng, D.; Chen, X. H.; Zhang, Y. Black Phosphorus Field-Effect Transistors. Nat. Nanotechnol. 2014, 9, 372.

(8) Zhu, Z.; Cai, X.; Yi, S.; Chen, J.; Dai, Y.; Niu, C.; Guo, Z.; Xie, M.; Liu, F.; Cho, J.-H.; 

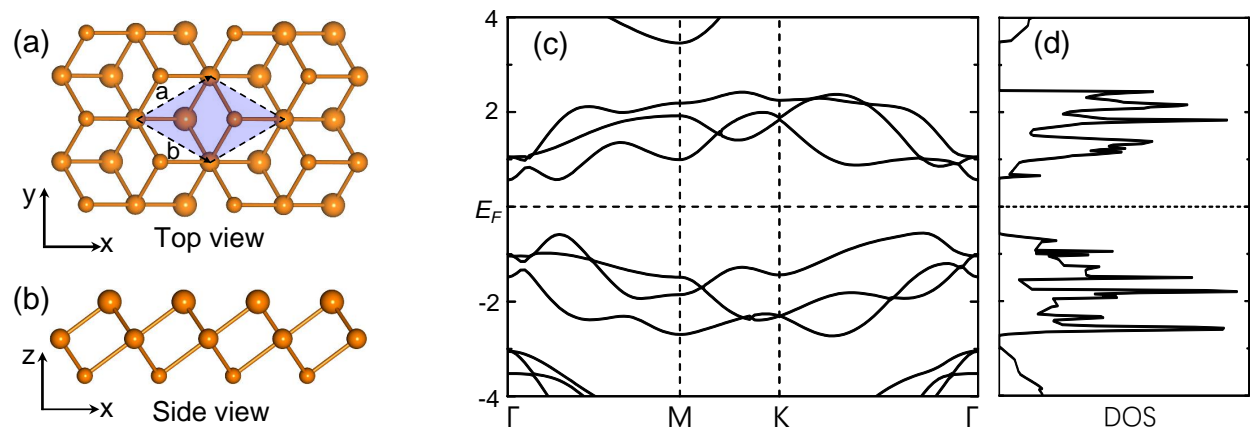

Figure 1: (a)(b) Top and side views of the optimized $\alpha$-Te monolayer structure. The primitive cell is indicated by the blue shading in the top view. $a$ and $b$ are the lattice vectors spanning the 2D lattice. (c)(d) Electronic band structure and density of states (DOS) of $\alpha$-Te at HSE06 level. The Fermi levels are set to zero.
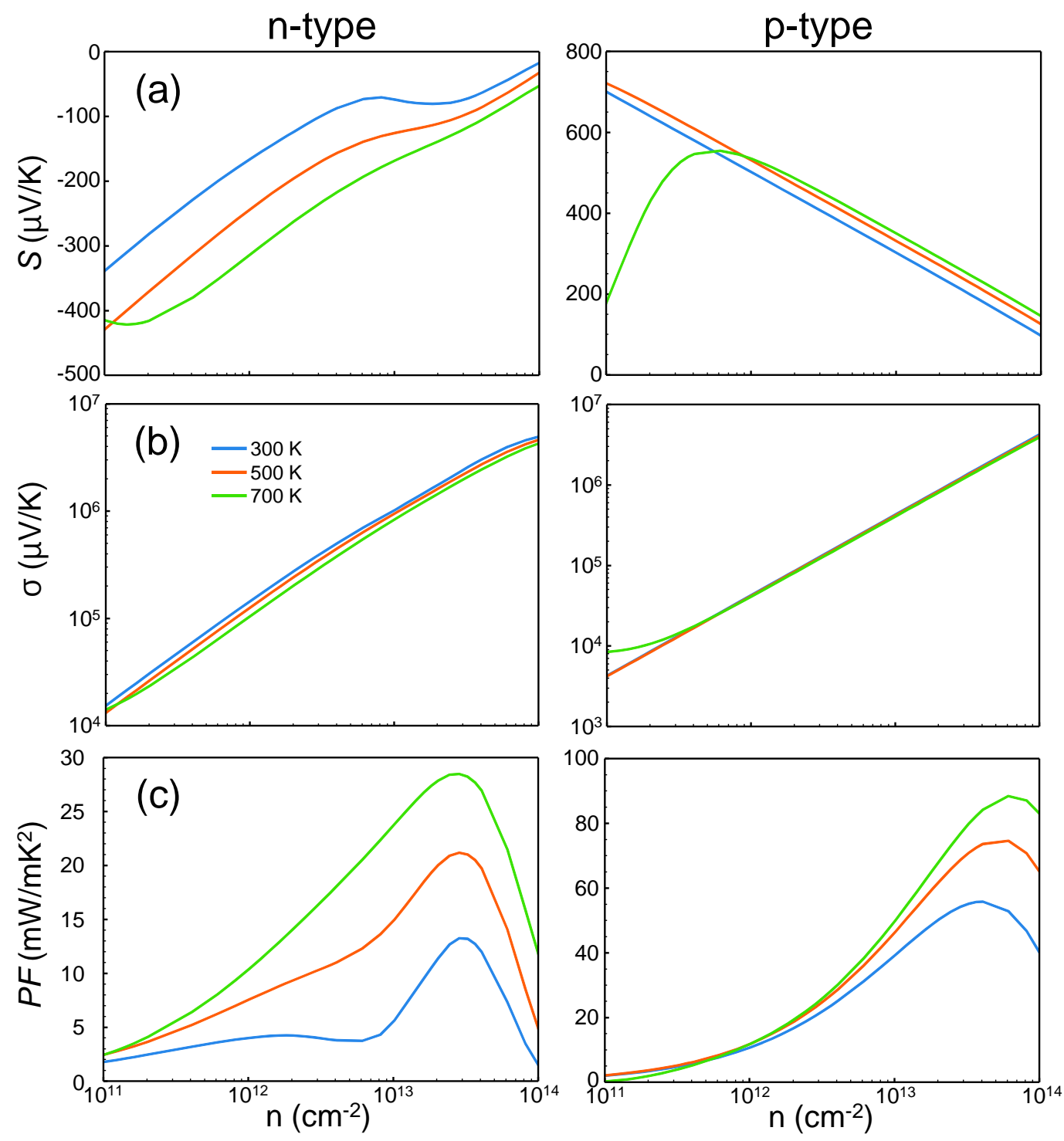

Figure 2: Temperature-dependent electronic transport coefficients. (a) Seebeck coefficient, (b) electrical conductivity, and (c) power factor $\left(S^{2} \sigma\right)$ of the $\alpha$-Te as a function of carrier concentration along $a$ or $b$ axis at 300, 500, and $700 \mathrm{~K}$. 

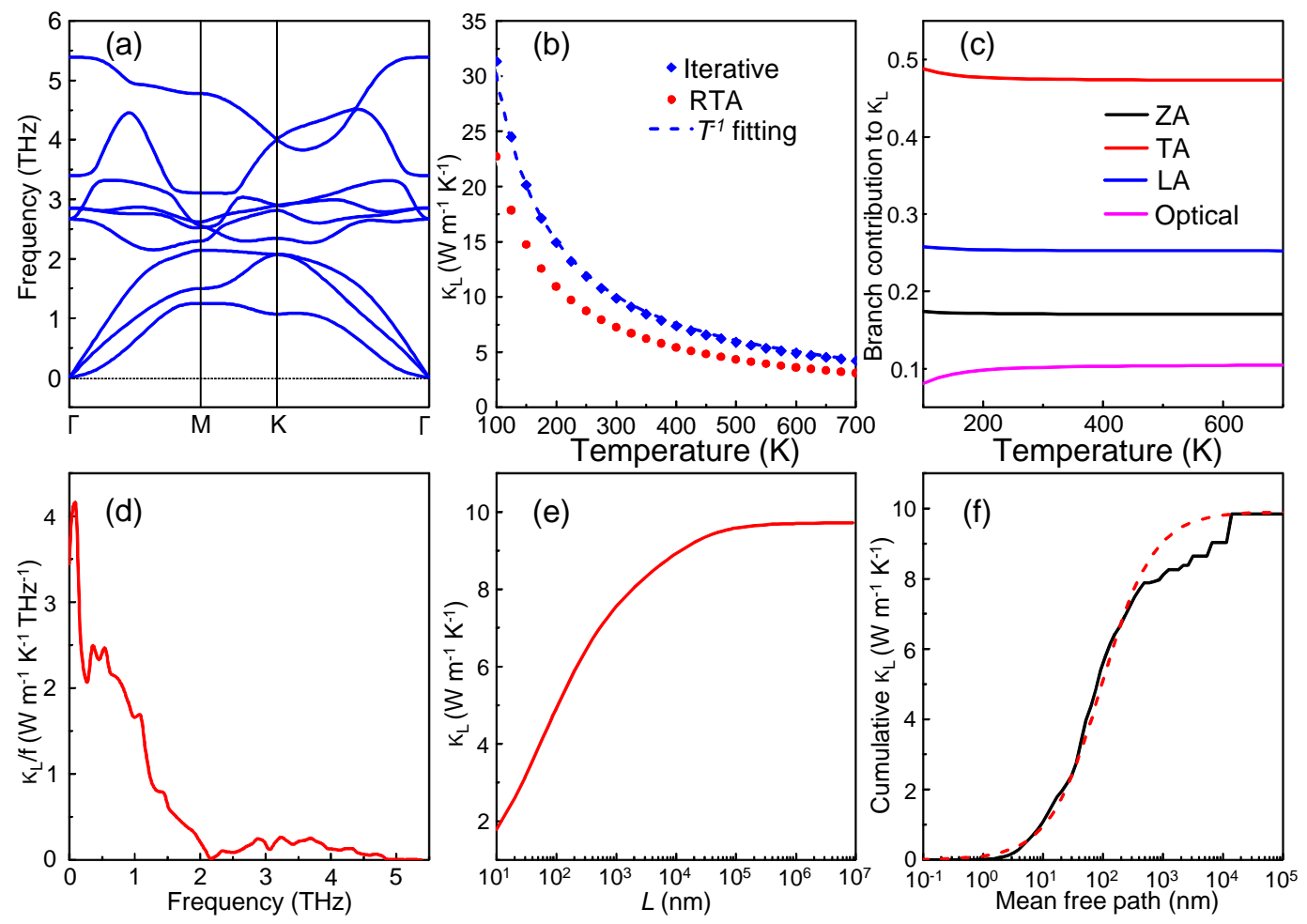

Figure 3: (a) Phonon spectra and DOS of $\alpha$-Te. (b) Intrinsic lattice thermal conductivity $\kappa_{L}$ as a function of temperature. Blue dashed line is $T^{-1}$ fitting. The values of relaxation time approximation (RTA) are also shown by red dots. (c) Normalized contributions to total $\kappa_{L}$ of phonon branches from 100 to $700 \mathrm{~K}$. (d) Frequency-resolved $\kappa_{L}$ at room temperature. (e) Room-temperature $\kappa_{L}$ as a function of sample size. (f) Cumulative $\kappa_{L}$ as a function of phonon mean free path (MFP) at room temperature. The fitted curve is plotted in a dashed line. 

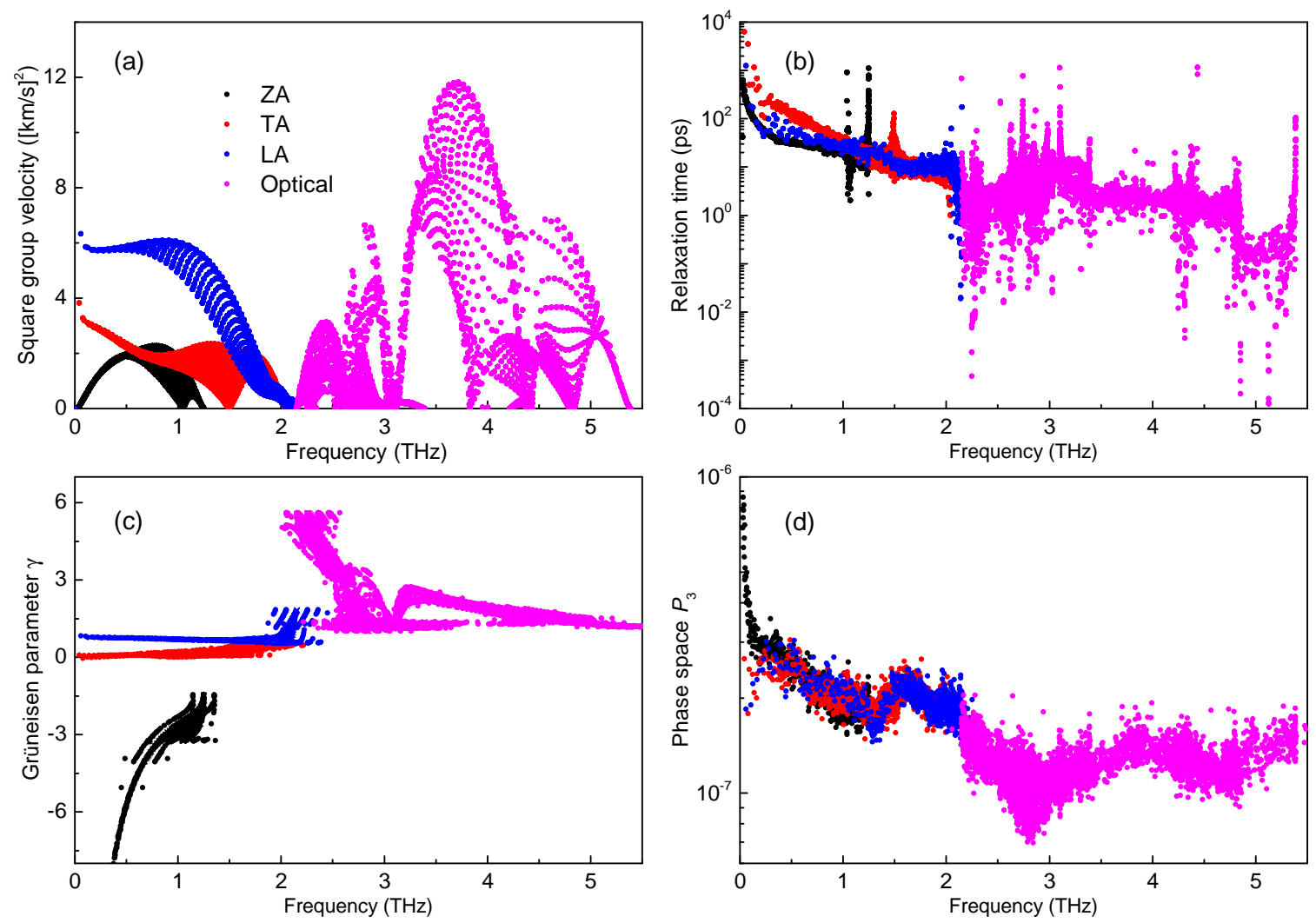

Figure 4: (a) The frequency dependent behavior of square group velocity at $300 \mathrm{~K}$ for $\alpha$-Te. All optical phonon branches are in purple. (b) Phonon relaxation time of ZA, TA, LA, and optical phonon branches as a function of frequency at room temperature. (c) Mode-Grüneisen parameter and (d) Phase-space volume $P_{3}$ for three-phonon scattering processes.
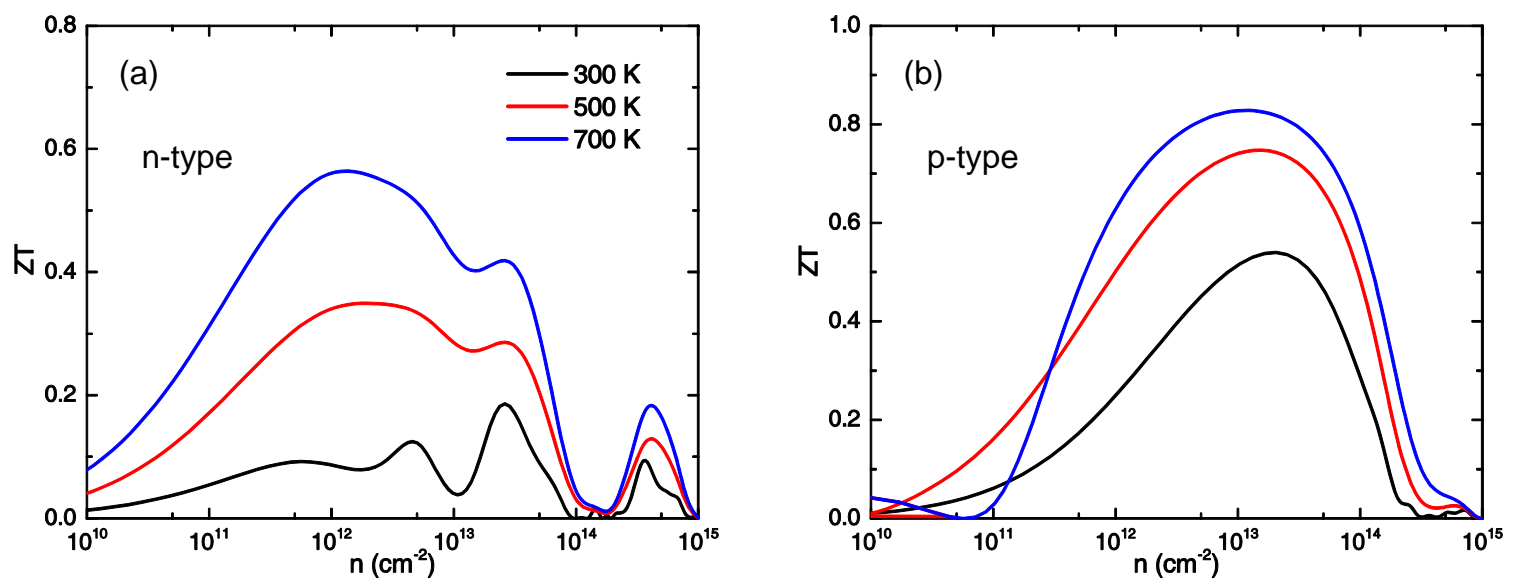

Figure 5: Calculated (a) n-type and (b) p-type $Z T$ values of monolayer $\alpha$-Te as a function of carrier concentration along $a$ or $b$ axis at 300,500 and $700 \mathrm{~K}$. 

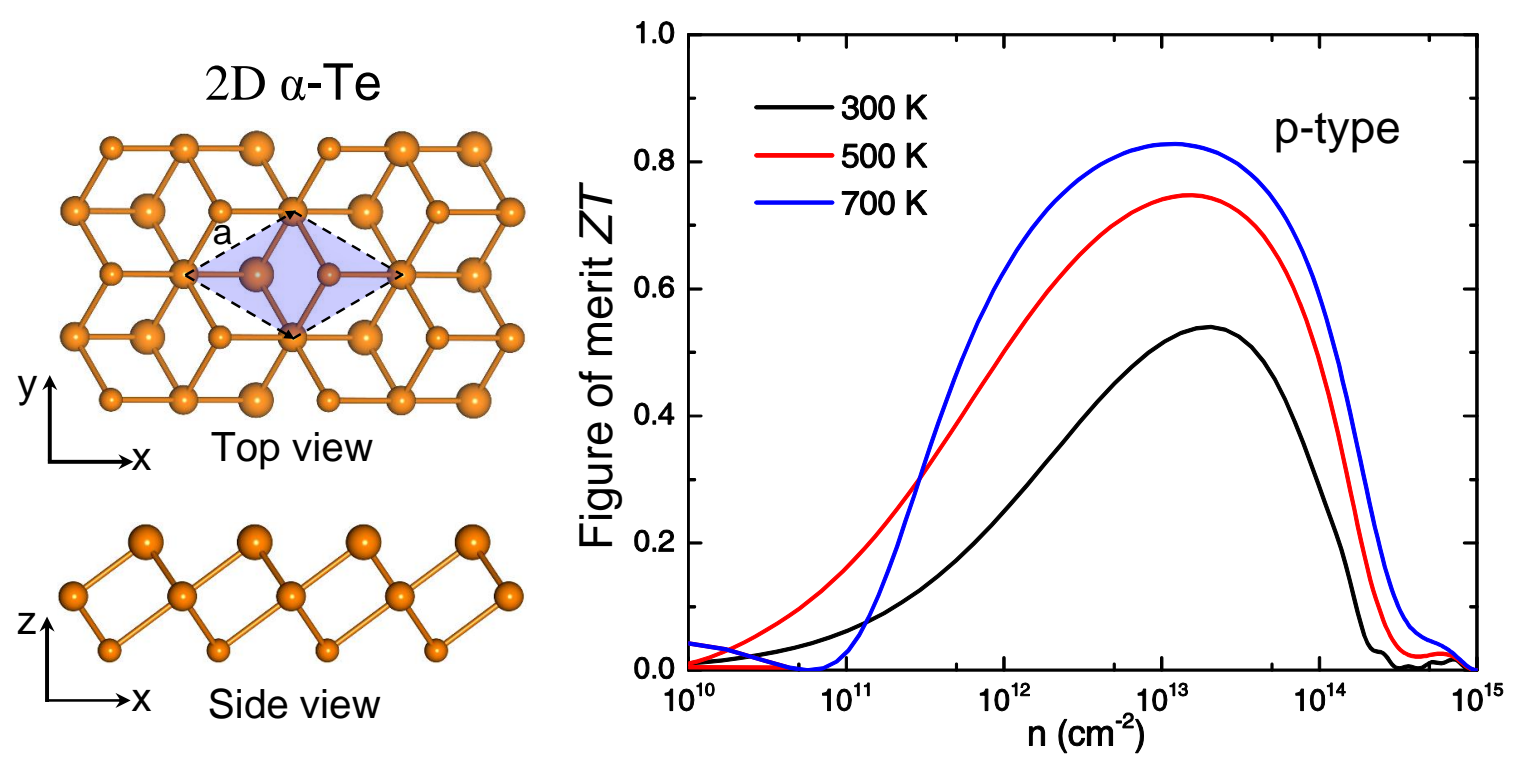

Table of Contents Graphic

Jia, Y.; Zhang, Z. Multivalency-Driven Formation of Te-Based Monolayer Materials: A Combined First-Principles and Experimental Study. Phys. Rev. Lett. 2017, 119, 106101.

(9) Huang, X.; Guan, J.; Lin, Z.; Liu, B.; Xing, S.; Wang, W.; Guo, J. Epitaxial Growth and Band Structure of Te Film on Graphene. Nano Lett. 2017, 17, 4619-4623.

(10) Wang, Y.; Qiu, G.; Wang, R.; Huang, S.; Wang, Q.; Liu, Y.; Du, Y.; Goddard, W. A.; Kim, M. J.; Xu, X.; Ye, P. D.; Wu, W. FieldEffect Transistors Made from SolutionGrown Two-Dimensional Tellurene. Nat. Electron. 2018, 1, 228.

(11) Du, Y.; Qiu, G.; Wang, Y.; Si, M.; $\mathrm{Xu}, \mathrm{X}$.; Wu, W.; Ye, P. D. One-Dimensional Van der Waals Material Tellurium: Raman Spectroscopy under Strain and MagnetoTransport. Nano Lett. 2017, 17, 3965-3973.

(12) Gao, Z.; Tao, F.; Ren, J. Unusually Low Thermal Conductivity of Atomically Thin 2D Tellurium. Nanoscale 2018, 10, 1299713003.

(13) Chen, J.; Dai, Y.; Ma, Y.; Dai, X.; Ho, W.; Xie, M. Ultrathin $\beta$-Tellurium Layers Grown on Highly Oriented Pyrolytic Graphite by
Molecular-Beam Epitaxy. Nanoscale 2017, 9, 15945-15948.

(14) Liu, Y.; Wu, W.; Goddard, W. A. Tellurium: Fast Electrical and Atomic Transport Along the Weak Interaction Direction. J. Am. Chem. Soc. 2018, 140, 550-553.

(15) Qiao, J.; Pan, Y.; Yang, F.; Wang, C.; Chai, Y.; Ji, W. Few-layer Tellurium: One-Dimensional-Like Layered Elementary Semiconductor with Striking Physical Properties. Sci. Bull. 2018, 63, 159 - 168.

(16) Sharma, S.; Singh, N.; Schwingenschlögl, U. Two-Dimensional Tellurene as Excellent Thermoelectric Material. ACS Appl. Energy Mater. 2018, 1, 1950-1954.

(17) Lin, S.; Li, W.; Chen, Z.; Shen, J.; Ge, B.; Pei, Y. Tellurium as a High-Performance Elemental Thermoelectric. Nat. Commnun. 2016, 7, 10287.

(18) Lee, S.; Esfarjani, K.; Luo, T.; Zhou, J.; Tian, Z.; Chen, G. Resonant Bonding Leads to Low Lattice Thermal Conductivity. Nat. Commun. 2014, 5, 3525.

(19) Tadano, T.; Gohda, Y.; Tsuneyuki, S. Impact of Rattlers on Thermal Conductivity of a 
Thermoelectric Clathrate: A First-Principles Study. Phys. Rev. Lett. 2015, 114, 095501.

(20) Jana, M. K.; Pal, K.; Waghmare, U. V.; Biswas, K. The Origin of Ultralow Thermal Conductivity in InTe: Lone-Pair-Induced Anharmonic Rattling. Angew. Chem., Int. Ed. 2016, 128, 7923-7927.

(21) Snyder, G. J.; Toberer, E. S. Complex Thermoelectric Materials. Nat. Mater. 2008, 7, 105-114.

(22) Liu, H.; Shi, X.; Xu, F.; Zhang, L.; Zhang, W.; Chen, L.; Li, Q.; Uher, C.; Day, T.; Snyder, G. J. Copper Ion LiquidLike Thermoelectrics. Nat. Mater. 2012, 11, 422 .

(23) Blöchl, P. E. Projector Augmented-Wave Method. Phys. Rev. B: Condens. Matter Mater. Phys. 1994, 50, 17953-17979.

(24) Kresse, G.; Joubert, D. From Ultrasoft Pseudopotentials to the Projector AugmentedWave Method. Phys. Rev. B: Condens. Matter Mater. Phys. 1999, 59, 1758-1775.

(25) Perdew, J. P.; Burke, K.; Ernzerhof, M. Generalized Gradient Approximation Made Simple. Phys. Rev. Lett. 1996, 77, 3865-3868.

(26) Heyd, J.; Scuseria, G. E.; Ernzerhof, M. Hybrid Functionals Based on a Screened Coulomb Potential. J. Chem. Phys. 2003, 118, 8207-8215.

(27) Grimme, S. Semiempirical GGA-Type Density Functional Constructed with a LongRange Dispersion Correction. J. Comput. Chem. 2006, 27, 1787-1799.

(28) Madsen, G. K.; Singh, D. J. BoltzTraP. A Code for Calculating Band-Structure Dependent Quantities. Comput. Phys. Commun. 2006, 175, 67-71.

(29) Chen, M. X.; Podloucky, R. Electronic Thermal Conductivity as Derived by Density Functional Theory. Phys. Rev. B: Condens. Matter Mater. Phys. 2013, 88, 045134.
(30) Togo, A.; Oba, F.; Tanaka, I. First-Principles Calculations of the Ferroelastic Transition Between Rutile-Type and $\mathrm{CaCl}_{2}$-Type $\mathrm{SiO}_{2}$ at High Pressures. Phys. Rev. B: Condens. Matter Mater. Phys. 2008, 78, 134106.

(31) Li, W.; Carrete, J.; Katcho, N. A.; Mingo, N. ShengBTE: A Solver of the Boltzmann Transport Equation for Phonons. Comput. Phys. Commun. 2014, 185, 1747.

(32) Lee, S.; Hippalgaonkar, K.; Yang, F.; Hong, J.; Ko, C.; Suh, J.; Liu, K.; Wang, K.; Urban, J. J.; Zhang, X.; Dames, C.; Hartnoll, S. A.; Delaire, O.; Wu, J. Anomalously Low Electronic Thermal Conductivity in Metallic Vanadium Dioxide. Science 2017, $355,371-374$.

(33) Crossno, J.; Shi, J. K.; Wang, K.; Liu, X.; Harzheim, A.; Lucas, A.; Sachdev, S.; Kim, P.; Taniguchi, T.; Watanabe, K.; Ohki, T. A.; Fong, K. C. Observation of the Dirac Fluid and the Breakdown of the Wiedemann-Franz Law in Graphene. Science 2016, 351, 1058-1061.

(34) Dutta, B.; Peltonen, J. T.; Antonenko, D. S.; Meschke, M.; Skvortsov, M. A.; Kubala, B.; König, J.; Winkelmann, C. B.; Courtois, H.; Pekola, J. P. Thermal Conductance of a Single-Electron Transistor. Phys. Rev. Lett. 2017, 119, 077701.

(35) Mosso, N.; Drechsler, U.; Menges, F.; Nirmalraj, P.; Karg, S.; Riel, H.; Gotsmann, B. Heat Transport through Atomic Contacts. Nat. Nanotechnol. 2017, 12, 430.

(36) Gao, Z.; Dong, X.; Li, N.; Ren, J. Novel Two-Dimensional Silicon Dioxide with inPlane Negative Poisson's Ratio. Nano Lett. 2017, 17, 772-777.

(37) Peng, H.; Kioussis, N.; Snyder, G. J. Elemental Tellurium as a Chiral $p$-Type Thermoelectric Material. Phys. Rev. B: Condens. Matter Mater. Phys. 2014, 89, 195206.

(38) Heremans, J. P.; Jovovic, V.; Toberer, E. S.; Saramat, A.; Kurosaki, K.; Charoenphakdee, A.; Yamanaka, S.; Snyder, G. J. En- 
hancement of Thermoelectric Efficiency in PbTe by Distortion of the Electronic Density of States. Science 2008, 321, 554-557.

(39) Hicks, L. D.; Dresselhaus, M. S. Effect of Quantum-Well Structures on the Thermoelectric Figure of Merit. Phys. Rev. B: Condens. Matter Mater. Phys. 1993, 47, 1272712731.

(40) Hicks, L. D.; Dresselhaus, M. S. Thermoelectric Figure of Merit of a OneDimensional Conductor. Phys. Rev. B: Condens. Matter Mater. Phys. 1993, 47, 1663116634.

(41) Mahan, G. D.; Sofo, J. O. The Best Thermoelectric. Proc. Natl. Acad. Sci. U. S. A. 1996, 93, 7436-7439.

(42) Kuroki, K.; Arita, R. "Pudding Mold" Band Drives Large Thermopower in $\mathrm{NaxCoO}_{2} . J$. Phys. Soc. Jpn. 2007, 76, 083707.

(43) Usui, H.; Suzuki, K.; Kuroki, K.; Nakano, S.; Kudo, K.; Nohara, M. Large Seebeck Effect in Electron-Doped $\mathrm{FeAs}_{2}$ Driven by a Quasi-One-Dimensional Pudding-MoldType Band. Phys. Rev. B: Condens. Matter Mater. Phys. 2013, 88, 075140.

(44) Ge, X.-J.; Qin, D.; Yao, K.-L.; Lü, J.-T. FirstPrinciples Study of Thermoelectric Transport Properties of Monolayer Gallium Chalcogenides. J. Phys. D, Appl. Phys. 2017, 50, 405301.

(45) Mi, X.-Y.; Yu, X.; Yao, K.-L.; Huang, X.; Yang, N.; L, J.-T. Enhancing the Thermoelectric Figure of Merit by Low-Dimensional Electrical Transport in Phonon-Glass Crystals. Nano Lett. 2015, 15, 5229-5234.

(46) Bilc, D. I.; Hautier, G.; Waroquiers, D.; Rignanese, G.-M.; Ghosez, P. Low-Dimensional Transport and Large Thermoelectric Power Factors in Bulk Semiconductors by Band Engineering of Highly Directional Electronic States. Phys. Rev. Lett. 2015, 114, 136601.
(47) Yang, J.; Liu, R.; Chen, Z.; Xi, L.; Yang, J.; Zhang, W.; Chen, L. Power Factor Enhancement in Light Valence Band p-Type Skutterudites. Appl. Phys. Lett. 2012, 101, 022101.

(48) Yang, J.; Qiu, P.; Liu, R.; Xi, L.; Zheng, S.; Zhang, W.; Chen, L.; Singh, D. J.; Yang, J. Trends in Electrical Transport of $p$-Type Skutterudites $R \mathrm{Fe}_{4} \mathrm{Sb}_{12}(R=\mathrm{Na}, \mathrm{K}, \mathrm{Ca}, \mathrm{Sr}$, $\mathrm{Ba}, \mathrm{La}, \mathrm{Ce}, \mathrm{Pr}, \mathrm{Yb}$ ) from First-Principles Calculations and Boltzmann Transport Theory. Phys. Rev. B: Condens. Matter Mater. Phys. 2011, 84, 235205.

(49) Yang, J.; Li, H.; Wu, T.; Zhang, W.; Chen, L.; Yang, J. Evaluation of Half-Heusler Compounds as Thermoelectric Materials Based on the Calculated Electrical Transport Properties. Adv. Funct. Mater. 2008, 18, 28802888 .

(50) Yang, J.; Xi, L.; Qiu, W.; Wu, L.; Shi, X.; Chen, L.; Yang, J.; Zhang, W.; Uher, C.; Singh, D. J. On the Tuning of Electrical and Thermal Transport in Thermoelectrics: an Integrated Theory-Experiment Perspective. NPJ. Comput. Mater. 2016, 2, 15015.

(51) Pei, Y.; Shi, X.; LaLonde, A.; Wang, H.; Chen, L.; Snyder, G. J. Convergence of Electronic Bands for High Performance Bulk Thermoelectrics. Nature 2011, 473, 66.

(52) Bilc, D. I.; Hautier, G.; Waroquiers, D.; Rignanese, G.-M.; Ghosez, P. Low-Dimensional Transport and Large Thermoelectric Power Factors in Bulk Semiconductors by Band Engineering of Highly Directional Electronic States. Phys. Rev. Lett. 2015, 114, 136601.

(53) He, J.; Amsler, M.; Xia, Y.; Naghavi, S. S.; Hegde, V. I.; Hao, S.; Goedecker, S.; Ozolinšs, V.; Wolverton, C. Ultralow Thermal Conductivity in Full Heusler Semiconductors. Phys. Rev. Lett. 2016, 117, 046602.

(54) Poncé, S.; Margine, E.; Verdi, C.; Giustino, F. EPW: Electron-phonon Coupling, Transport and Superconducting Properties Using Maximally Localized Wannier Functions. Comput. Phys. Commun. 2016, 209, 116-133. 
(55) Qiao, J.; Kong, X.; Hu, Z.-X.; Yang, F.; Ji, W. High-Mobility Transport Anisotropy and Linear Dichroism in Few-Layer Black Phosphorus. Nat. Commun. 2014, 5, 4475.

(56) Wang, F. Q.; Zhang, S.; Yu, J.; Wang, Q. Thermoelectric Properties of Single-Layered SnSe Sheet. Nanoscale 2015, 7, 1596215970.

(57) Lindsay, L.; Broido, D. A.; Reinecke, T. L. First-Principles Determination of Ultrahigh Thermal Conductivity of Boron Arsenide: A Competitor for Diamond? Phys. Rev. Lett. 2013, 111, 025901.

(58) Gao, Z.; Li, N.; Li, B. Heat Conduction and Energy Diffusion in Momentum-Conserving One-Dimensional Full-Lattice Ding-a-Ling Model. Phys. Rev. E 2016, 93, 022102.

(59) Gao, Z.; Li, N.; Li, B. Stretch Diffusion and Heat Conduction in One-Dimensional Nonlinear Lattices. Phys. Rev. E 2016, 93, 032130.

(60) Seol, J. H.; Jo, I.; Moore, A. L.; Lindsay, L.; Aitken, Z. H.; Pettes, M. T.; Li, X.; Yao, Z.; Huang, R.; Broido, D.; Mingo, N.; Ruoff, R. S.; Shi, L. Two-Dimensional Phonon Transport in Supported Graphene. Science 2010, 328, 213-216.

(61) Balandin, A. A. Thermal Properties of Graphene and Nanostructured Carbon Materials. Nat. Mater. 2011, 10, 569-581.

(62) Lindsay, L.; Li, W.; Carrete, J.; Mingo, N.; Broido, D. A.; Reinecke, T. L. Phonon Thermal Transport in Strained and Unstrained Graphene from First Principles. Phys. Rev. B: Condens. Matter Mater. Phys. 2014, 89, 155426.

(63) Lindsay, L.; Broido, D. A.; Mingo, N. Flexural Phonons and Thermal Transport in Graphene. Phys. Rev. B: Condens. Matter Mater. Phys. 2010, 82, 115427.

(64) Peng, B.; Zhang, H.; Shao, H.; Xu, Y.; Ni, G.; Zhang, R.; Zhu, H. Phonon Transport Properties of Two-Dimensional Group-IV Materials from $a b$ initio Calculations. Phys. Rev.
B: Condens. Matter Mater. Phys. 2016, 94, 245420.

(65) Ma, J.; Chen, Y.; Han, Z.; Li, W. Strong Anisotropic Thermal Conductivity of Monolayer $\mathrm{WTe}_{2}$. 2D Mater. 2016, 3, 045010.

(66) Lindsay, L.; Broido, D. Three-Phonon Phase Space and Lattice Thermal Conductivity in Semiconductors. Journal of Physics: Condensed Matter 2008, 20, 165209.

(67) Lv, B.; Lan, Y.; Wang, X.; Zhang, Q.; Hu, Y.; Jacobson, A. J.; Broido, D.; Chen, G.; Ren, Z.; Chu, C.-W. Experimental Study of the Proposed Super-Thermal-Conductor: BAs. Appl. Phys. Lett. 2015, 106, 074105. 